\title{
Some Challenges of the Development of Constitutive Equations of Semicrystalline Polymers for Structural Calculations
}

\author{
Y. Meimon ${ }^{1}$ \\ 1 Institut français du pétrole, 1 et 4, avenue de Bois-Préau, 92852 Rueil-Malmaison Cedex - France \\ e-mail: yves.meimon@ifp.fr
}

\begin{abstract}
Résumé - Enjeux du développement de lois de comportement de polymères semi-cristallins pour le calcul de structure - L'utilisation des polymères semi-cristallins à usage structural se généralise dans l'industrie. Ces polymères solides ont un comportement mécanique compliqué à appréhender, dépendant fortement du temps et de la température, et sensible en particulier au vieillissement chimique, un point délicat quand il s'agit d'évaluer la durée de vie des structures. Le développement de lois de comportement capables de décrire l'évolution de la mémoire des polymères semi-cristallins sous les sollicitations imposées aux structures, et leur insertion dans un code de calcul par la méthode des éléments finis sont des moyens privilégiés pour améliorer le dimensionnement des structures.

La qualité de ces techniques de calcul complexes doit être assurée par une méthodologie adaptée au développement de lois de comportement des polymères semi-cristallins. Celle-ci peut être imagée par les étapes du « cycle de vie » d'un modèle : de la conception du modèle à la validation sur expérimentations de structures types représentatives du fonctionnement de l'ouvrage. Ces étapes sont commentées en détail en utilisant une nouvelle modélisation du comportement des polymères semi-cristallins.
\end{abstract}

Mots-clés : polymères semi-cristallins, lois de comportement, méthode aux éléments finis, méthodologie de développement de lois de comportement, validation pour le calcul de structure.

\footnotetext{
Abstract - Some Challenges of the Development of Constitutive Equations of Semicrystalline Polymers for Structural Calculations - The use of semicrystalline polymers as structural polymers is spreading in the industry. The mechanical behaviour of these polymers is difficult to grasp, highly dependent on time and temperature, and particularly sensitive to chemical ageing, an important point when evaluating the time life of structures. The development of constitutive equations which can describe the evolution of the mechanical memory of semicrystalline polymers and their insertion in a computer code employing the finite element method are preferential means for improving structural design.

The quality of these complex computation techniques must be assured by a methodology adapted to the development of constitutive equations of semicrystalline polymers. This methodology may be pictured by the steps of the "life cycle" of a model: from the design of the model up to the validation on "typical structures" representing the structure in operation. These steps are detailed in the paper by using, as an example, a new model of the behaviour of semicrystalline polymers.

Keywords: semicrystalline polymers, constitutive equations, finite element method, methodology of development of constitutive equations, validation for structure calculation.
} 


\section{INTRODUCTION}

The use of structural polymers, generally semicrystalline polymers (SCP), a powerful impetus for innovation in difficult environmental conditions, is spreading in the industry. Examples include pump bodies, seal barriers and flexible metal pipes for oil transport, water and gas supply pipes, fuel tanks for automobiles. Yet, the mechanical behaviour of SCPs is difficult to grasp, highly dependent on time and temperature, and particularly sensitive to chemical ageing, which embrittles the material and shortens the life of the structures.

The development of constitutive equations, which can describe the evolution of the memory of the polymers (anelastic, irreversible deformation, strain hardening) under the loads imposed on the structures, and their insertion in a computer code employing the finite element method are preferential means for improving structural design under service loads. This involves using the complex computation techniques offered by advances in information processing, and now applicable with office computers. The quality of the modelling procedures is hence extremely important for the use of these techniques. It must be assured by a methodology adapted to the development of the constitutive equations of polymers.

\section{THE NEED FOR A METHODOLOGY FOR DEVELOPING CONSTITUTIVE EQUATIONS OF POLYMERS}

\subsection{Complexity of Polymer Behaviour in Structures}

The example of flexible flowlines for offshore oil and gas transport (Fig. 1a) demonstrates the complexity of the behaviour of polymers in structures. A flexible pipe is made up of many layers of mobile metal armatures, and the sealing function is performed by layers of thermoplastic polymers (Fig. 1b). A pipe must be able to withstand high internal pressure (up to $100 \mathrm{MPa}$ ) of a fluid of variable acidity, at temperatures up to $130^{\circ} \mathrm{C}$. Depending on the service conditions, a choice is made among SCPs like polyethylene (PE), polyamide 11 (PA11) and polyvinylidene fluoride (PVF2). These materials are not strictly impermeable: under the pipe service pressure, gas may dissolve and saturate the material. Designed for use over several decades, these structures are liable to sudden operating interruptions, resulting in a sharp drop in pressure in the pipes. The gas present in the polymer is forced to diffuse outside the material and could damage it. Figures $2 \mathrm{a}$ and $2 \mathrm{~b}$ show structural damage to low-density PE samples saturated with methane. It is caused by more or less rapid decompression (Jarrin et al., 1994). Depending on the speed of the decompression, bubbles or cracks are observed at the end of the tests. With some polymers, decompression may also produce foam. An understanding of this mechanism obviously demands detailed consideration of the microstructure of the SCP and of its chemical affinity with the fluid. This is achieved by coupling the viscoplastic deformation of the material with fluid diffusion and the change in temperature. At the theoretical level, these coupled mechanisms may be approached, at a mesoscopic scale, via the thermodynamics of open systems (Coussy, 1991). In certain conditions (essentially, isotropy and small strains hypotheses), the following incremental equations can be derived, chiefly linking stress $\sigma$, strain $\varepsilon$, fluid pressure $p$, external input of fluid mass $m$, entropy $s$ and temperature $T$ :

$$
\begin{aligned}
& \dot{\sigma}=C\left(\dot{\varepsilon}-\dot{\varepsilon}^{p}\right)-b \dot{p} \mathbf{1}-3 \alpha K \dot{T} \mathbf{1} \\
& \dot{p}=M\left[-b\left(\dot{\varepsilon}_{v}-\dot{\varepsilon}_{v}^{p}\right)+\left(\dot{m} / \rho_{0}^{f}-\beta \dot{\varepsilon}_{v}^{p}\right)\right]+3 \alpha_{m} M \dot{T} \\
& \dot{s}=\dot{m} s_{0}+3 \alpha K\left(\dot{\varepsilon}_{v}-\dot{\varepsilon}_{v}^{p}\right)-3 \alpha_{m} \dot{p}+C_{\varepsilon}^{0} \dot{T} / T_{0}
\end{aligned}
$$
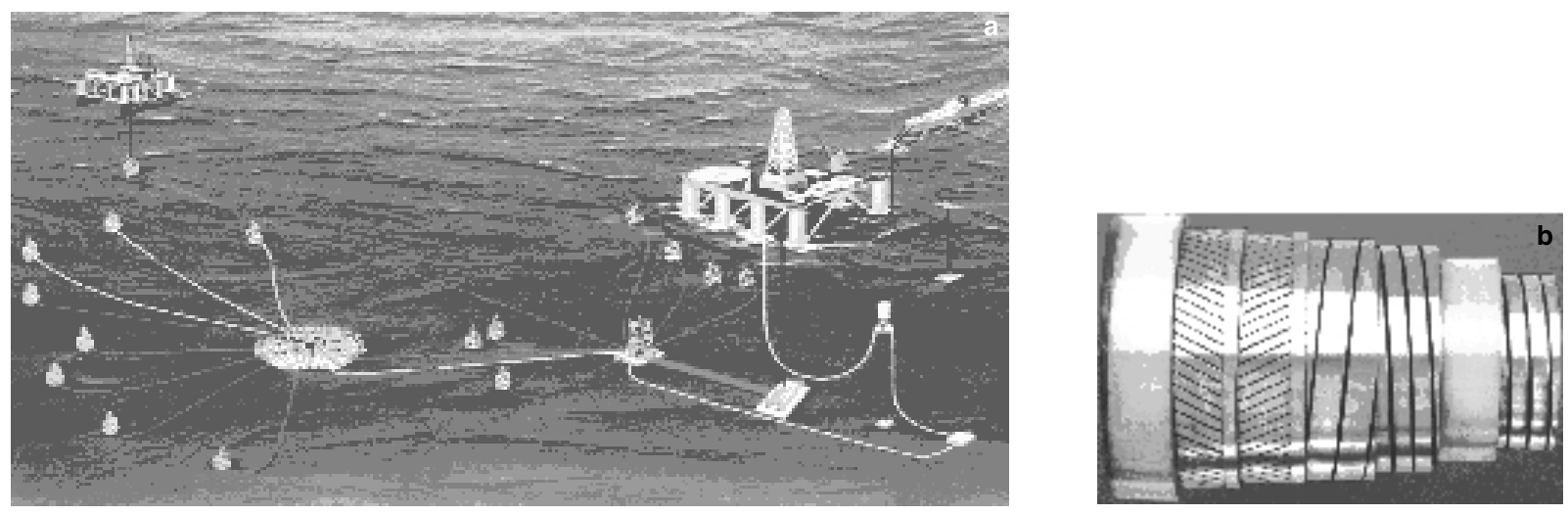

Figure 1

Offshore oil and gas production.

(a) Offshore production platform with flexible risers and flowlines.

(b) Cutaway view of a Coflexip riser. 


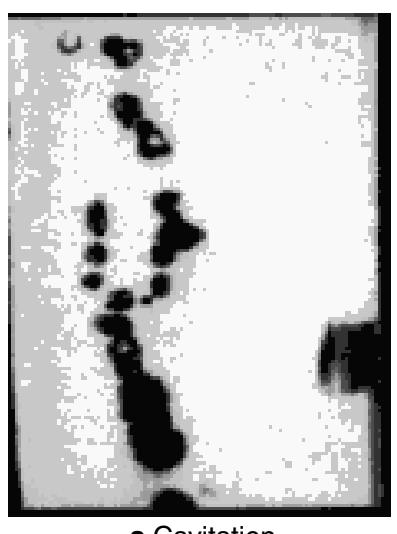

a Cavitation

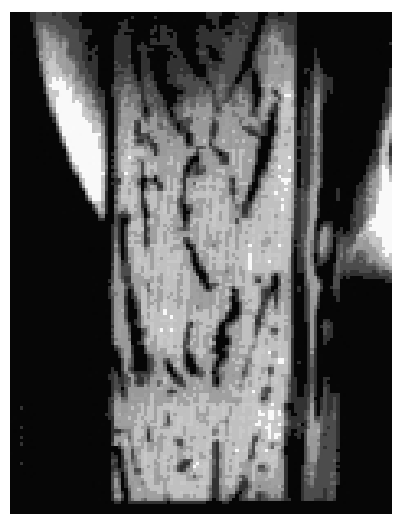

b Cracking
Figure 2

Damage observed in low-density PE under pressure, saturated with methane during (a) slow, (b) rapid decompression (Jarrin et al., 1994).

Here $C, b, K, M, \alpha, \alpha_{m}, \beta, \rho_{0}^{f}$ and $C_{\varepsilon}^{0}$ are constant parameters of the medium, $T_{0}, s_{0}$ are respectively the reference temperature and entropy of the medium, and $\varepsilon_{v}$ is the volumetric strain of the material. These equations reveal the importance of the constitutive equation of the material. It is represented by the change in anelastic deformation $\varepsilon^{p}$ and, in particular, due to the isotropy hypothesis, by the change in the corresponding volumetric strain $\varepsilon_{v}^{p}$, which is directly involved in the equation of state of fluid pressure and in the equation of state of entropy of the system. In the case of conduction, Fourier equation allows to evaluate the entropy variation $s-m s_{0}$. Added to these equations is the evolution of the mass of the fluid saturating the material and exchanged with the exterior: this reveals to be a good subject of investigation for SCPs in petroleum applications.

Thus to calculate and dimension correctly, indeed optimise, structures comprising SCPs in interaction with the environment, it is crucial to describe accurately the mechanisms governing their deformation via constitutive equations. The complexity of the geometry of the structures and of the loads applied entails the use of numerical methods, like the finite element method, to calculate the deformation at any point. A spatial discretisation is carried out with simple geometrical elements, in which the local behaviour of the material is calculated, using the constitutive equation.

From the foregoing example, it is clear that a phenomenological approach is only truly advantageous if it relies on the observation of the structure of the material and of the mechanisms of its deformation, i.e. on relations between structure and mechanical properties. It requires coupling analyses at several scales: from microscopic scale, at which the material is heterogeneous, for the deformation of the macromolecules in different states (amorphous, crystalline), to the macroscopic scale of the laboratory specimen test. This may lead to sophisticated constitutive equations, for which a quality approach must be applied before they can be used for structural calculations.

\section{2 "Life Cycle" of a Constitutive Equation}

Figure 3 illustrates a methodology, already used positively in other areas (Meimon, 1990), which can be adopted to develop constitutive equations of polymers. It is similar to the functional analysis method developed for the design cycle of a product containing elastomers (Coupard, 1997). Before industrial application, i.e. the use of the equation for structural calculations, many steps must be completed:

- conception of the model, associated with the industrial need which sets the specification, with the theoretical and experimental state of the art, and with the intuition of the formalisms used to describe the pattern of the deformation mechanisms identified;

- validation of the constitutive equation "on homogeneous paths", typical of laboratory tests on specimens, is indispensable to guarantee the credibility of the model. This step serves to verify that the equation responds as intended by the designer, and to test its limits in not necessarily foreseeable situations, or for paths that are unfeasible today in the laboratory, but which could be obtained in the structural calculation;

- validation of the constitutive equation on "typical structure", representing the structure in operation, is the final step of the process. In fact, at any given time, no equation can fully account for the complex reality. Hence it is important to be sure that the constitutive equation, which is guaranteed at the theoretical level and for simple homogeneous paths, functions correctly for the structure concerned.

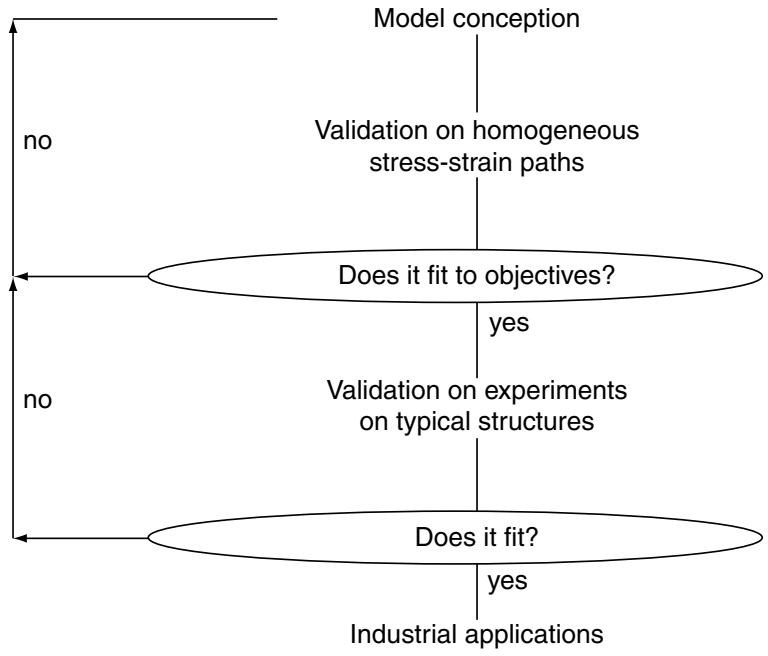

Figure 3

"Life cycle" of a constitutive equation. 
At each validation step, the designer may be led to review the formulation of his model to improve the response to the problem at hand. This makes the overall results of the "life cycle" a process of validation, which could represent the quality insurance file of the constitutive equation, and which may prove useful at the time of industrial application.

\section{DEVELOPMENT OF CONSTITUTIVE EQUATIONS FOR STRUCTURAL CALCULATIONS}

\subsection{Methodology for Developing Constitutive Equations}

The conception of a constitutive equation can be seen as the result of four different modelling operations:

- the physical model relates the changes in macroscopic variables to those of the microstructural deformation. This requires using experimental resources that yield reliable data at different scales, in order to substantiate the hypotheses of the model. For SCPs, the task is difficult due to the heterogeneity of the microstructure, but feasible: a simple specimen loading test (generally in uniaxial tensile loading) is combined with a sophisticated means of investigation to examine the deformation of the microstructure up to failure. This approach was followed by Dahoun (1992) to analyse and model the plastic behaviour and deformation textures of several SCPs, for example, by using wide-angle X-rays, at temperatures between the glass-transition temperature and crystalline fusion. Similarly, based on observations on high-density PE (tunnel effect electron microscope, small-angle X-ray diffusion for lamellar deformation, wide-angle X-ray diffraction for crystalline deformation) during deformation, Argon (1997) presented a fairly realistic model for large deformations, according to which the evolution of the texture of the material is the result of continuous shear at constant volume of the lamellae, combined, to a lesser degree, with amorphous interlamellar shear. However, the main difficulty is to make these micromechanical models usable for structural calculations by the finite element method. Another approach, using this type of observation, and giving rise to a model that can be easily integrated in a structural computation code, is considered below: the model is defined, directly, at a mesoscopic scale, at which the material can be considered to consist of homogeneous phases;

- the mechanical model formalises the physical model in a coherent thermodynamic framework. The thermodynamics of irreversible processes of open systems (for example, of porous systems (Coussy, 1991)) is a framework which may be appropriate to polymers in structures if their interaction with fluids (water, gas, petroleum fluids) is to be described. As a consequence, an incremental equation will be used linking the temperature, the stress state and the strain state of the material by using internal variables which characterise the work-hardening of the material. This leads generally to a mechanical constitutive equation developed in the framework of elasticity, viscoplasticity and damage theory. This equation may be rather complicated in order to account for cyclic loading and, more generally, non-monotonic loading;

- the numerical model allows correct integration of the mechanical non-linear model developed, for which there is generally no analytical solution. Effective algorithms have been developed for elastoviscoplastic models and for viscoelastic models, even when several plastic mechanisms are coupled to describe the anelastic material behaviour. However, special care should be taken for integrating large strains that may occur in polymers;

- the computer code is the tangible tool representing the constitutive equation. Note that the object-oriented programming method helps to guarantee its quality and upgradability today.

For SCPs, the formulated constitutive equation is always non-linear, using internal variables to describe the state of strain hardening of the material. Accordingly, the mechanical parameters are of two types: those which can be determined directly by analysing standard tests (for example, elastic constants of the material), and those which govern the changes in the internal variables and must be determined by numerical fitting of the equation to an experimental curve.

Hence the development of the constitutive equation must be accompanied by a procedure for determining the parameters, which must be made objective and rapid. This procedure must enable a user unfamiliar with the model to obtain a realistic set of parameters for the material concerned within a reasonable time, based on tests and/or a minimum of science and expertise stored in a software. This expertise enables the user of the equation to identify an initial set of parameters and to determine the final set by following a strategy proposed by the designer. Software of this type is available (Pilvin, 1988; Meimon, 1990) but is not yet specialised for polymers.

\subsection{Example of a Multiscale Approach for Semicrystalline Polymers}

The microstructure of SCPs consists of an amorphous phase and a crystalline phase consisting of stacks of lamellae. These are often organised in the form of spherolites, as shown in Figure 4, using the scanning electron microscopy (SEM), for the PVF2 microstructure of a sample cut off in a sheet made up by quenching in cold water: obviously, the two phases are deeply imbricated. The physicochemical characterisation of SCPs tends to imply that the amorphous 


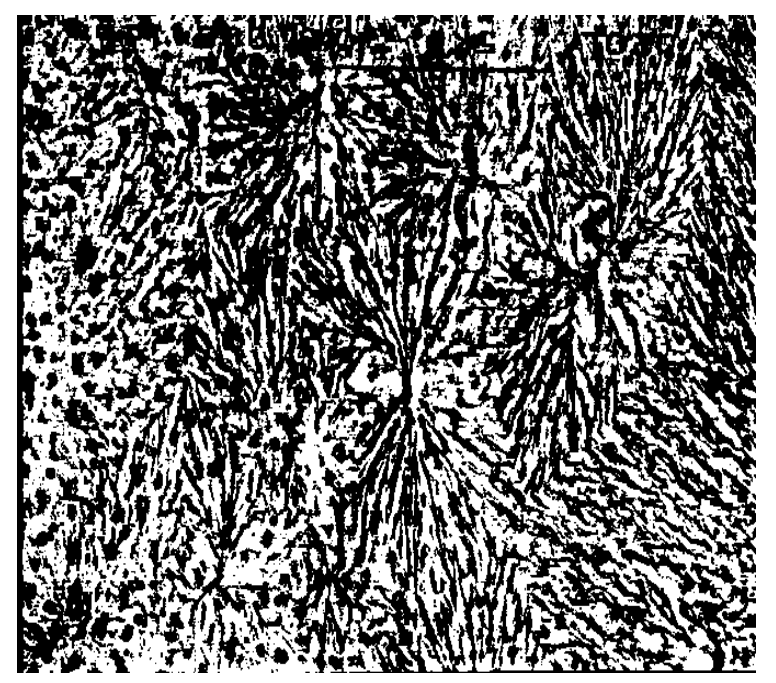

Figure 4

Spherolitic structure of a PVF2 (Dal Maso, 1992).

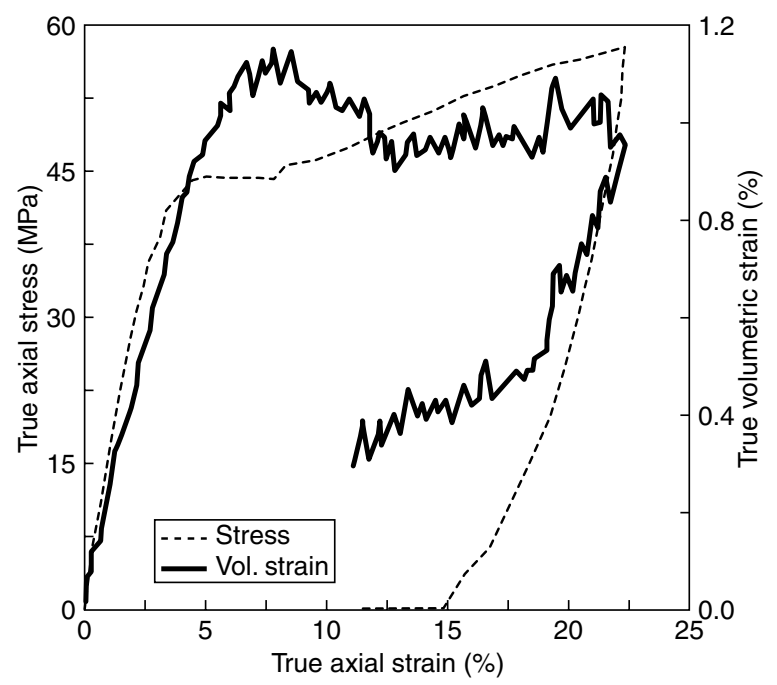

Figure 5

Uniaxial tensile test $\left(\dot{\varepsilon}=10^{-3} / \mathrm{s}\right.$ ) with measurement of change in volume on unplasticised PA11, below $T_{g}$ (Marchal, 1996). phase has a heterogeneous structure due to the presence of crystallites, which locally reduce molecular mobility. Struik (1987) investigated this point. According to his "extended glass-transition" model, a temperature interval exists, around the glass-transition temperature $\left(T_{g}\right)$, in which a part of the amorphous phase is in a rubbery state, with the rest in a vitreous state. For PA11, the heterogeneity of the amorphous phase is also supported by the fact that the deuteration (i.e. change of hydrogen atoms by deuterium) is only possible on a part of the amorphous phase (probably the most mobile), regardless of the means employed (Wu and Siesler, 1997). An analysis of the texture of the material by wide-angle Xray diffraction, carried out at different axial deformation rates, leads to the conclusion that a third phase may exist, which is neither pure amorphous nor crystallites (Jolly, 2000). As a first approximation, one can assume a splitting of the amorphous phase into a "bonded (or confined) amorphous" part of which the mobility is reduced (by the crystallites) and a "free amorphous" part corresponding to the portion of the chains free to motion.

On the mechanical point of view, the spherolitic microstructure of the material and the interaction of the amorphous and crystalline phases result in a complicated evolution of the variation in volume, even on the simple uniaxial tensile path. Figure 5 shows the evolution of the volumetric strain, measured by using a laser optical technique, during a tensile test on PA11 (Marchal, 1996). The volumetric strain first rises, then tends to decrease when the draw hook is reached. A tendency to contraction is then obtained while the true axial stress continues to increase.

The effect of the mean stress must also be considered. Figure 6 compares the results of a simple compression test and a triaxial compression test performed at a $10 \mathrm{MPa}$ confining pressure, on unplasticised PA11. Qualitatively, one can note a tendency to expansion of the material in simple compression, and also that the confinement contains the volumetric strain and increases the strength of the material. The influence of the mean stress on the mechanical behaviour of the material can also be observed when comparing compression, torsion and tensile tests, as Castagnet (1998) made for PVF2.

Besides, it is also well known that the presence of amorphous material in SCP gives rise to mechanical behaviour that is highly sensitive to the loading rate applied and to temperature, as well as high recovery after unloading.

These overall results serve to suggest a two-phase mesoscopic model (Meimon and Cangémi, 1999) of the mechanical behaviour of SCPs (named Vispol), directly usable in a structural calculation code by the finite element method. The microstructure (heterogeneous) is represented by two phases, homogeneous at mesoscopic scale, in close interaction (Fig. 7). The first phase consists of a structure of crystalline lamellae surrounded by amorphous material in a confined state, and behaves like a "solid". The second consists exclusively of free amorphous material which can be compared to a rubbery phase. Due to the relative softness of this phase, it is assumed that the shear stress in it is negligible with respect to the shear stress in the "solid" phase. Hence, as a first approximation, it is assumed that it behaves like a "fluid" without viscosity, when the strains remain small or finite.

Since SCPs have a spherolitic structure, these two phases, "solid" and "fluid", are closely imbricated and it is essential to describe their interaction in order to define a stress-strain equation on a homogenised elementary volume. The same 

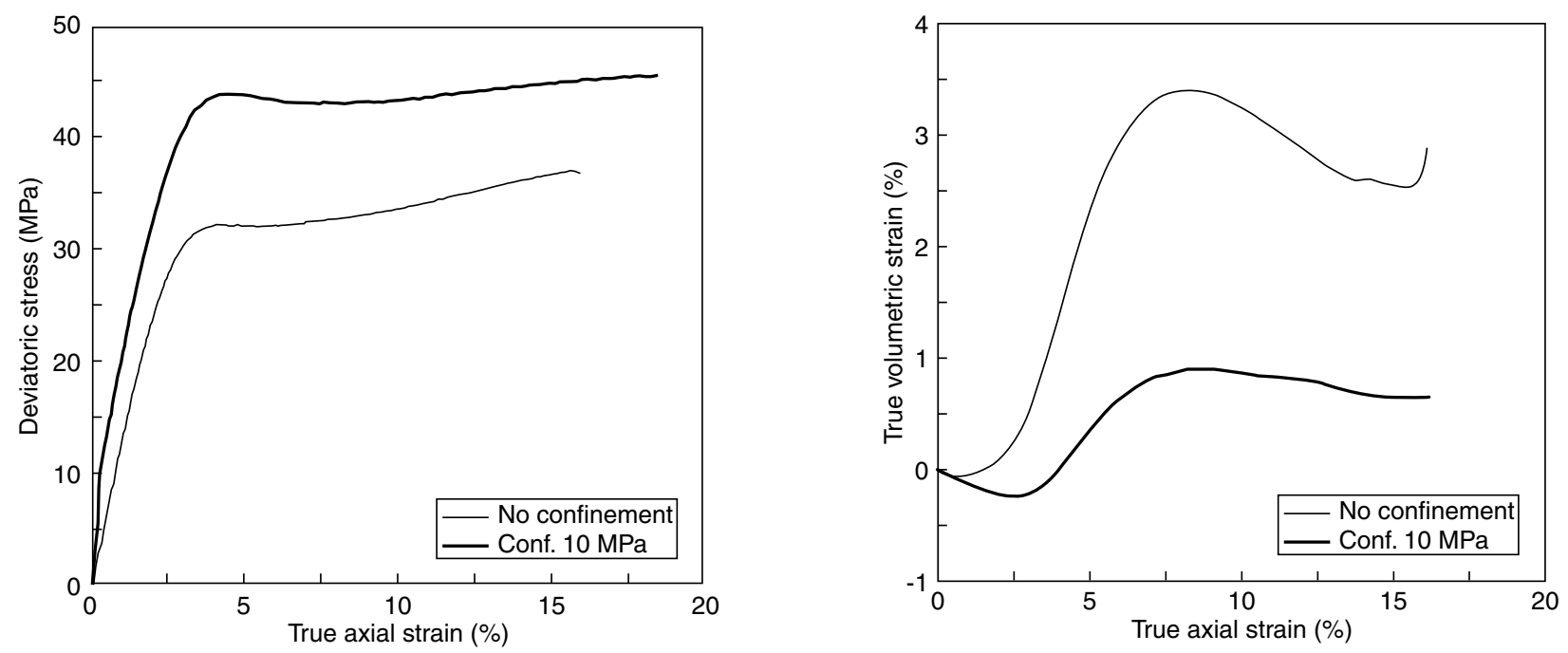

Figure 6

Triaxial compression of PA11 ( $\left.\dot{\varepsilon}=10^{-4} / \mathrm{s}\right)$ (Vincké, 1997).

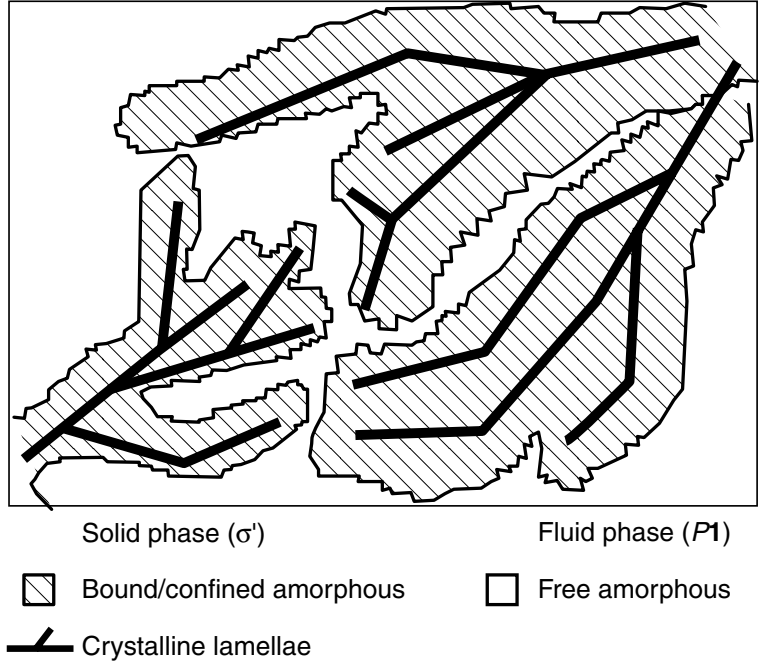

Figure 7

Vispol model. Representation of the microstructure in two homogeneous phases at mesoscopic scale.

method as for geomaterials may be applied by considering that the volume occupied by the "fluid" (i.e. the free amorphous content $\Phi)$ is similar to the porosity of a geomaterial and that the "solid" is similar to the solid skeleton of the geomaterial. According to the thermodynamics of open systems (Coussy, 1991) and assuming isothermal small strains and material isotropy at macroscopic scale, the following equations can be established:

$$
\begin{gathered}
\sigma=\sigma^{\prime}-B P \mathbf{1} \\
m / \rho_{0}=P / M_{a}+B \varepsilon_{v}+\left(\beta_{a}-B\right) \varepsilon_{v}^{p}
\end{gathered}
$$

where $\sigma$ is the total stress in the material, $\sigma$ ' the stress in the "solid", $P$ the pressure in the "fluid" and $\mathbf{1}$ the identity tensor. In Equation (5), $m$ is the external input of "fluid" mass to the system, $\varepsilon_{v}$ is the volumetric strain of the material, $\varepsilon_{v}^{p}$ is the irreversible volumetric strain of the material and $\rho_{0}$ is the density of the free amorphous phase. The material properties $B, \mathrm{~b}_{a}$ and $M_{a}$ are linked to the free amorphous content $\Phi$ in the material and, normally, depend on the temperature.

Equation (5) describes the effect of a "fluid" mass input, $m$, to the material: here, this can be interesting for modelling the effect of the loss of plasticiser additive in certain SCPs (this additive being considered as the "fluid" type). In the standard case, where $m=0$, Equation (5) reduces to:

$$
P / M_{a}=-B \varepsilon_{v}-\left(\beta_{a}-B\right) \varepsilon_{v}^{p}
$$

Hence, from Equation (6), the pressure $P$ of the free amorphous phase is essentially inferred from the behaviour of the (crystalline + confined amorphous) phase: it rises or decreases according to whether the variation in volume of this phase decreases or rises. Thus, depending on its quantity present in the material ( $B$ and $\beta_{a}$ tend to zero if $\Phi$ tends to zero), the free amorphous phase plays the role of "damping" the deformation imposed on the (crystalline + confined amorphous) phase which, by virtue of its structure (Figs. 4 and 7), displays rather brittle behaviour.

In a first attempt to achieve the constitutive equation and due to the analogy with geomaterials, the behaviour of the (crystalline + confined amorphous) phase is represented by an elastoviscoplastic formalism with a plasticity mechanism, initially developed for soils. Details can be found in Aubry et al. (1985). In this model, the strain hardening is isotropic and described by means of $\varepsilon_{v}^{p}$ and $\varepsilon_{d}^{p}$ (the deviatoric plastic deformation). A viscosity damage mechanism may be used 

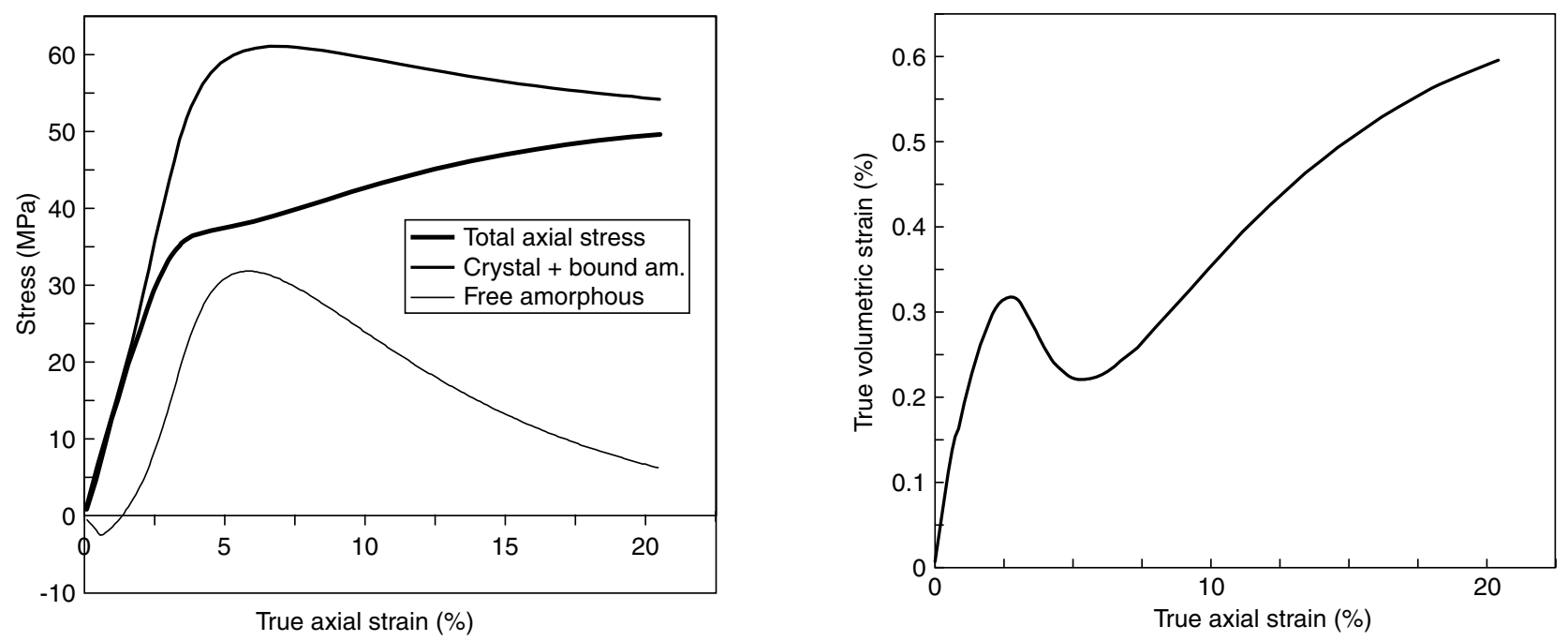

Figure 8

Vispol 1.0 model. Contribution of each phase in a uniaxial tensile test.

to account for diffuse microcracking due to dilatancy. Perzyna's approach is used, with the assumption that the plastic load surface and the failure mechanism in this phase can be represented by a Cam-Clay surface. This implies the existence of an internal friction angle of the (crystalline + confined amorphous) phase, which makes the mean stress influence the material behaviour. A plastic flow rule serves to describe the tendency to dilatancy or to contractancy, according to the value of the ratio (deviatoric stress / mean stress).

The functioning of the model, isothermal for the time being, is shown in Figure 8, where the contribution of each phase can be observed, according to Equation (6), for a monotonic tensile axial loading at constant strain rate $\left(10^{-4} / \mathrm{s}\right)$. Figure 9 , also on the uniaxial tensile path, shows that for a material like PA11, this type of flow can reproduce the draw hook and the effect of the axial strain rate. At the numerical level, the computation algorithm used locally to integrate this two-phase model proves to be relatively simple. It has been installed in a structural calculation code using the finite element method. While these first results are potentially encouraging, this type of model must nevertheless be fully validated before industrial application.

\section{VALIDATION ON HOMOGENEOUS PATHS}

\subsection{Principle}

The validation step on homogeneous paths in stresses and strains is particularly useful with polymers for which many constitutive equations are developed from uniaxial tensile tests, in which only the axial stress and strain are measured. It helps to substantiate the hypotheses made, to better

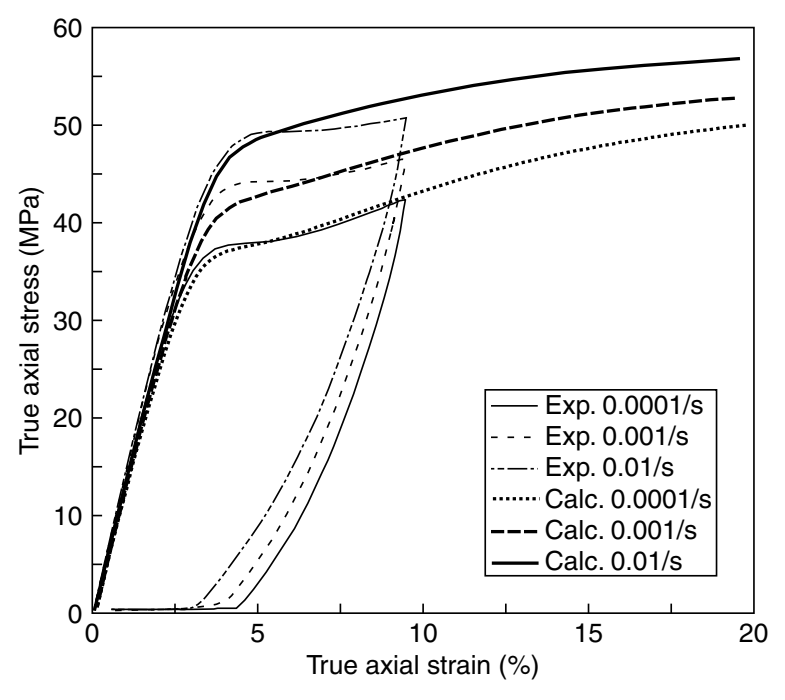

Figure 9

Vispol 1.0 model. Reproduction of uniaxial tensile tests performed at different axial strain rates.

understand the constitutive equation, and to identify its range of validity. In practice, it should be verified that:

- the constitutive equation correctly reproduces the laboratory tests. A distinction must be drawn between "fitting loading paths and sequences", on which the mechanical parameters of the constitutive equation have been determined, and "verification loading paths and sequences", on which it is validated. The latter are paths, which are feasible in the laboratory, and are typical of the functioning of the structure. For example, if uniaxial tensile 


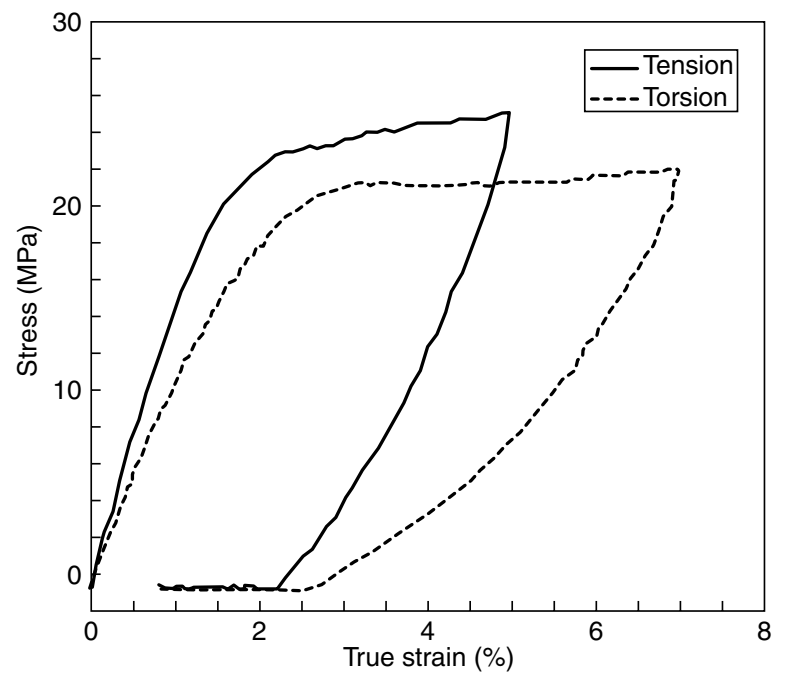

Figure 10

Tensile-torsion path on a PA11 pipe (Marchal, 1996).

tests at controlled axial strain rates are used to determine the parameters of an elastoviscoplastic equation, the equation could be verified with creep or relaxation tests, possibly on the same path. The best verification should be performed on multiaxial paths (G'Sell, 1995). For example, Figure 10 shows a test on a PA11 pipe in which a monotonic tensile load at constant strain rate is combined with torsion, also at constant strain rate, with a rate ratio of 0.36 (Marchal, 1996). These tests must be high-quality tests, i.e. both repeatable (in a given laboratory) and reproducible (by other laboratories). The latter condition is not always easy to satisfy since measurements, like that of the change in volume of the material, are difficult. Videometric test facilities (G'Sell et al., 1992) appear to be advantageous for this type of study, including at small deformations;

- the constitutive equation is "robust". It is essential to analyse the sensitivity of the response of the constitutive equation to disturbances in the mechanical parameters and the numerical parameters used in the integration algorithm. An excessive variation in the response of the equation for a small change in a parameter could make the parameter difficult to determine, and hence make the constitutive equation impractical;

- the constitutive equation yields plausible results on theoretical paths, which are today difficult to reproduce in the laboratory.

\subsection{Standardisation of Model Validation}

Validation on homogeneous paths is excessively timeconsuming to identify "failure diagnoses" of the constitutive equation, which could lead to its modifications and hence to the repetition of the validation procedure. This is easier if a software is available to standardise its performance. This purpose is fulfilled by the Valops software developed at IFP for the numerical validation of mechanical constitutive equations of polymers in the solid state (Ferrer and Meimon, 1995). The aim of Valops, for a given material, is to help to provide a diagnosis for the use of a constitutive equation on a given load path.

A path is characterised by load directions in the stressstrain space to which the temperature and the pressure of a fluid diffusing through the material can be added. It is composed of loading sequences, which are possible loads on the path. For example, the uniaxial tensile path activates the three directions 11, 22 and 33 with the condition on the stresses: $\sigma_{22}=\sigma_{33}=0$. It includes monotonic loadings at constant strain rate, non-monotonic loadings (imposed strain and/or stress cycles), creep, relaxation, etc. It is immediately clear that a constitutive equation can only be valid on this path, even for a very simple, monotonic loading with a controlled axial strain rate, if it correctly reproduces the axial stress as well as the transverse strains (or the change in volume). This definition consequently implies the necessity to standardise the tests for validation on homogeneous paths. The tests, selected according to the content of the measurements and their repeatability, can be collected in a data bank: this was done in the BdePol bank (Ferrer, 1997) for tests on PA11 including uniaxial tensile and torsion paths and a multiaxial tensile-torsion path (Marchal, 1996).

The Valops computation module allows the integration of rheological models, as they are used in a finite element calculation code. The object-oriented programming of the software allows the very simple introduction of a new model.

The establishment of an objective diagnosis is a delicate feature of the process. The evaluation of the differences between calculations and experiments must first be standardised, and then interpreted. For each variable, these deviations should be calculated at the measurement points as a function of time. Figures $11 \mathrm{a}$ and $11 \mathrm{~b}$ compare the prediction of a simplified bilinear constitutive equation for a monotonic loading on the uniaxial tensile path on PA11: the deviation is naturally very small for the control variable which is the axial strain, whereas for the axial stress, the deviation is large at the beginning of the test (in this case, the experimental "zero" was not perfect), returning to a value conforming more closely to the visual impression furnished by the stress-strain curve. This evaluation method is more reliable than the direct comparison of the difference between the stress-strain curves. In fact, one can imagine the case of two calculation-experiment curves, plotted from measured variables (for example, the axial stress and the transverse strain in a uniaxial tensile test), partly merged: they could give the illusion of good agreement of the model with the experiment, whereas the "identical" points could correspond to different times (i.e. different axial strains)! 

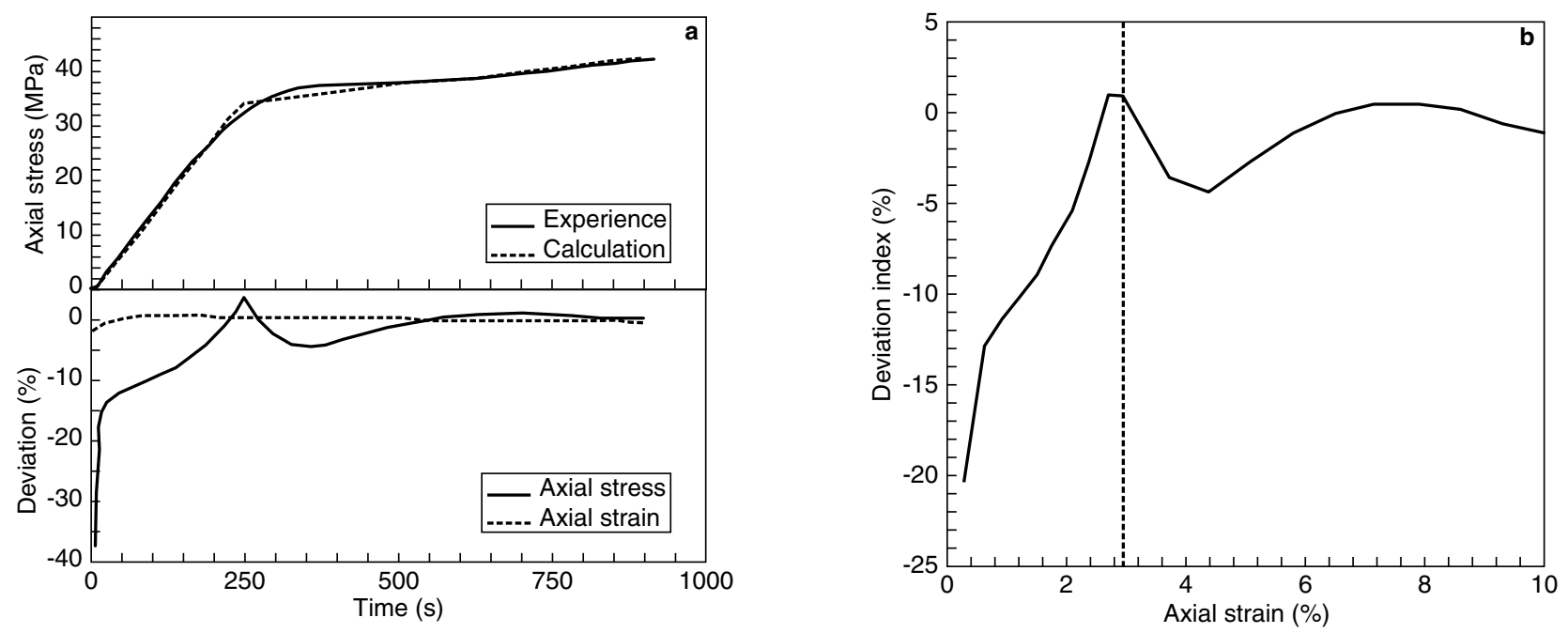

Figure 11

Valops: principle of evaluation of calculation-experiment deviations (monotonic loading on uniaxial tensile path).

(a) Axial stress and deviation as a function of time.

(b) Deviation index.

By a series of averaging operations on the differences obtained for each variable in all the available tests, it is possible to define a synthetic "deviation index" which is the total difference (defined in \%) between the equation and the experiment over a given interval of a reference variable. For example, on the uniaxial tensile path, and for a set of loadings including different rates, creep, relaxation, etc., a result like the one shown in Figure $11 \mathrm{~b}$ would indicate a maximum index of $5 \%$ between $3 \%$ and $10 \%$ axial deformation. It is up to the user to judge whether this is satisfactory for the application concerned or whether the formulation of the constitutive equation needs to be reviewed.

This diagnosis is clearly open-ended because it depends on the tests performed and on the quality of the measurements in the interval concerned of the reference variable. Valops helps update this diagnosis at any time in accordance with the enrichment of the test bank. Similarly, this method can be used to compare two constitutive equations or variants thereof. Thus the software, which is flexible and user-friendly, can be used as a "constitutive equation test bench" for polymers.

\section{VALIDATION ON TYPICAL STRUCTURES}

\subsection{Specification for Experiments on Typical Structure}

This step involves developing the method of use of the constitutive equation for the structure concerned, by using the finite element method. To do this, it is advantageous to exploit the typical-structure concept, which roughly represents the pair made up of the structure (geometry) and its operating or service conditions (loading, interaction with the environment). This enables the validation of the constitutive equation on clearly controlled and properly instrumented experiments conducted in a structural-testing laboratory.

Returning to the flexible pipe example, Figure 12 shows a simple experiment to ascertain the ability of an equation to reproduce the creep behaviour of a polymer sheath between the metal armatures, under the action of the pressure of a hot fluid. The experiment meets a precise specification, thereby helping to minimise the sources of experimental uncertainty:

- the geometry and boundary conditions are clearly defined and fully reproducible by a finite element calculation: the sheath is a circular pellet enclosed laterally in an indeformable metal box; it can creep in a circular hole made in the upper metal pellet, which is also circular; a pressure chamber is used to apply a uniform pressure to the sheath; the overall system is in a controlled-temperature enclosure; hence this is an axisymmetrical structure;

- the mechanical behaviour of the materials is clearly identified: this chiefly means the behaviour of the polymer, for which variable loadings (including creep) were carried out at different temperatures on the uniaxial tensile path; a sufficient number of tests was performed to determine the parameters of a constitutive equation and to validate it;

- the loading program is simple and easily reproducible by calculation;

- the measurements of the behaviour of the structure are repeatable. Overall measurements (creep) must be mixed with local measurements (material damage): for example, creep height of the sheath in the hole over time and deformed state of the sheath at the end of the test. 


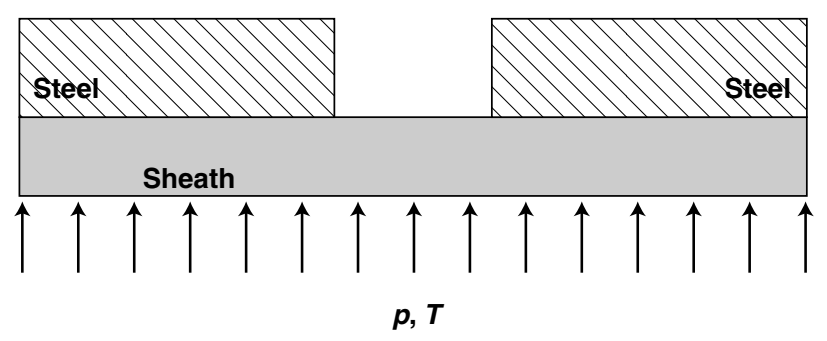

Figure 12

Example of typical structure for creep of a polymer sheath between metal armatures of a flexible pipe.

\subsection{Use of the Constitutive Equation in a Finite Element Calculation Program}

For a structural calculation with polymers, a methodological study should be carried out on the following points:

- the effect of the discretisation parameters: refinement of the mesh, particularly near the loadings and the large deformation zones; incrementation of the loading which must comply with the formulation of the constitutive equation. It is important to check the latter point, even if most of the major codes available use algorithms of automatic incrementation of the loading with refinement if necessary. As a rule, the loading step refinement criterion is not included in the constitutive equation. It is based on the increase in the number of iterations for the calculation of an increment. In fact, with some constitutive equations (elastoplasticity with kinematic hardening, DNLR models (Loukil, 1996)), it is possible to obtain a rapid overall convergence of the calculation with an inaccurate local integration of the behaviour when the loading increments are too large;

- the robustness of the numerical algorithm: convergence of calculations, particularly for large localised deformations with damage to the material;

- a study of sensitivity to variations in the parameters of the constitutive equation: this supplements the corresponding study carried out to validate the equation on homogeneous paths. Yet, its purpose is different: its aim is to identify, for the typical structure concerned, the parameters of the equation, which must be determined accurately. Some parameters may in fact have some influence on a tensile path and prove to be "inactive" for calculating the typical structure concerned. In the case of the Vispol model, inserted in Fondof, an IFP finite element code, Figure 13a shows the deformed geometry of a plane deformation calculation of $2 \mathrm{~mm}$ penetration of a $20 \mathrm{~mm}$ PA11 sheath by a flat $10 \mathrm{~mm}$ metal tool. Figure $13 \mathrm{~b}$ shows the sensitivity of the response of the equation on the uniaxial tensile path for two parameters: the elastic modulus which
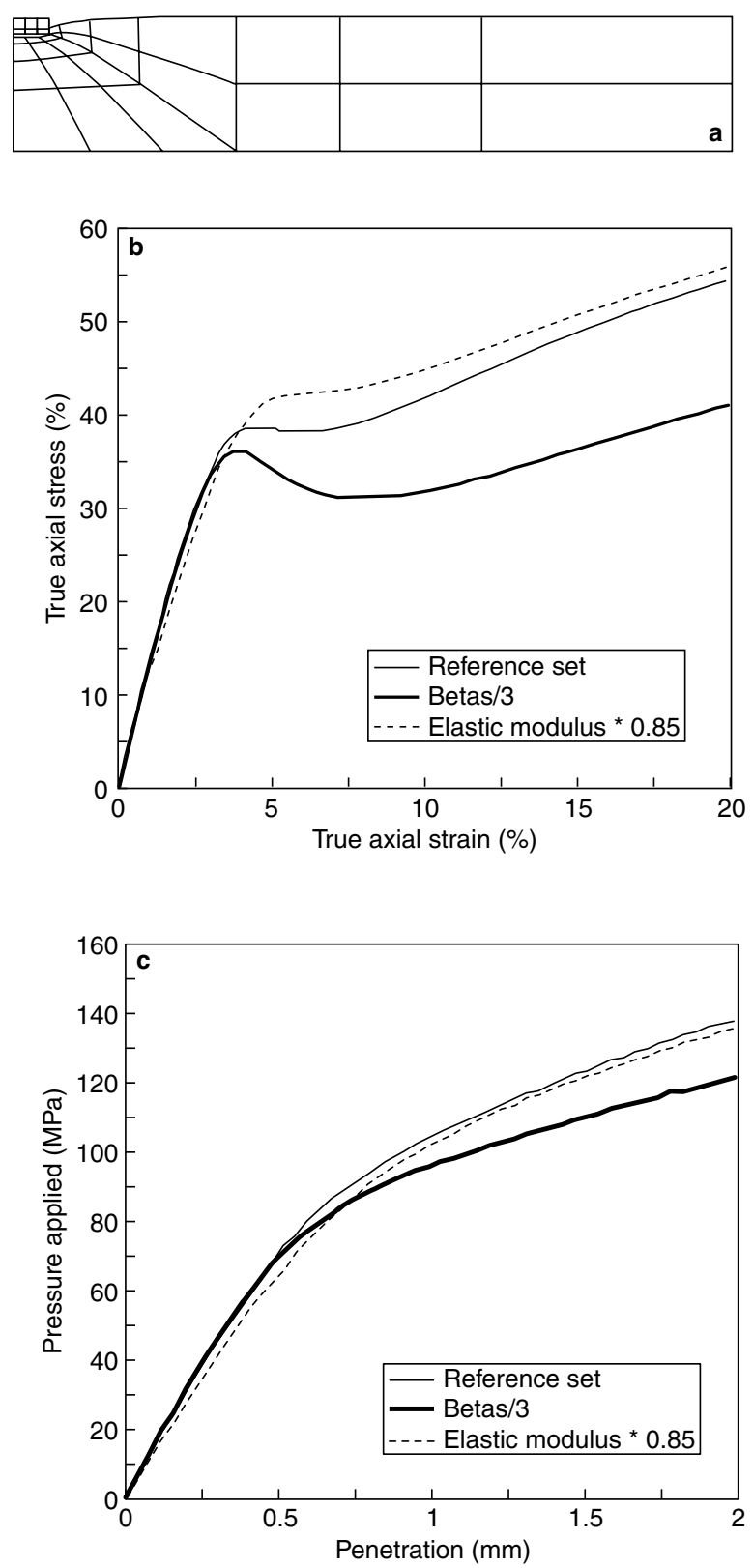

Figure 13

Vispol model: analysis of sensitivity to parameters for penetration of a steel punch in a PA11 $20 \mathrm{~mm}$ sheath.

(a) Deformed mesh for $2 \mathrm{~mm}$ penetration.

(b) Sensitivity of the response of the constitutive equation on homogeneous uniaxial tensile path.

(c) Sensitivity of the response of the constitutive equation for calculation of the punch by the finite element method.

has been decreased by $15 \%$, the parameter $\beta_{s}$ (it governs the isotropic hardening and the plasticity threshold) which has been divided by a factor of 3 . These parameters have a clear influence, particularly $\beta$, from the draw hook. Figure $13 \mathrm{c}$ shows the sensitivity of the response of the 
equation for the finite element calculation. The influence of the elastic modulus becomes weak for a penetration of more than $0.7 \mathrm{~mm}$, while a variation of $15 \%$ is found in displacement at the start of loading (as expected because the material firstly behaves elasticly). The influence of $\beta_{s}$ is only significant from $0.5 \mathrm{~mm}$ penetration when the material clearly plasticises. One could, for example, draw the conclusion that a precise determination of the elastic modulus is not essential to faithfully reproduce the behaviour at large penetrations.

In some cases, particularly when the constitutive equation is used for very different loads from those feasible in the laboratory on homogeneous paths (for example high-speed dynamic loadings) on which it has been validated, the prediction of the behaviour of the typical structure may prove to be very imperfect. This normally entails a return to the design step. In fact, modelling by finite elements could be used to optimise the set of mechanical parameters, which is accordingly only valid for the typical structure concerned (inverse methods).

\section{CONCLUSIONS}

Polymers may display highly non-linear behaviour for the range of loads applied in the structures. With SCPs, which are widely used in structures for difficult environmental conditions, macroscopic behaviour is strongly dependent on the microstructural morphology. Hence it is necessary to continue to:

- expand the knowledge of the mechanisms without hesitating to use sophisticated experimental resources combining the analysis of macroscopic deformation with the analysis of microstructural deformation. These resources are available and should, for example, help to develop physical models at a mesoscopic scale, at which the material can be considered homogeneous. Substantial progress still needs to be achieved to analyse the coupling mechanisms of mechanical behaviour with fluid diffusion across the polymers;

- develop three-dimensional constitutive equations by following a rigorous methodology: experimental methods provide access to the three-dimensional deformation of the material, and theoretical tools and software help guarantee the quality of the equations. These constitutive equations can be applied in finite element calculation codes for industrial use, provided they are validated on typical structures, depicting the geometry and operation of real structures.

\section{REFERENCES}

Argon, A.S. (1997) Morphological Mechanisms and Kinetics of Large-Strain Plastic Deformation and Evolution of Texture in Semicrystalline Polymers. J. of Computer-Aided Mat. Design, 4, 75-98.
Aubry, D., Kodaissi, E. and Meimon, Y. (1985) A Viscoplastic Constitutive Equation for Clays Including a Damage Law. 5th Int. Conf. on Num. Methods in Geomechanics, Nagoya, Japan.

Castagnet, S. (1998) Comportement mécanique du PVDF : compétition entre cavitation et écoulement visqueux. Thesis, University of Poitiers.

Coupard, A. (1997) Nouvelle approche dans le choix des élastomères. Mechanical Engineering of Rubbers, INPL, 223255.

Coussy, O. (1991) Mécanique des milieux poreux, Technip Ed., Paris.

Dahoun, A. (1992) Comportement plastique et textures de déformation des polymères semi-cristallins en traction uniaxiale et en cisaillement simple. Thesis, INPL.

Dal Maso, F. (1992) Influence du vieillissement thermique sur la morphologie et les propriétés du poly(fluore de vinylidène) $\left(\mathrm{PVF}_{2}\right)$. Thesis, University Pierre et Marie Curie.

Ferrer, G. (1997) Validation des lois de comportement pour les polymères solides. Banque d'essais mécaniques BdePol : matériau PA11. IFP Report.

Ferrer, G. and Meimon, Y. (1995) Validation des lois de comportement pour les polymères solides. Logiciel Valops : conception et spécifications. IFP Report.

G'Sell, C., Hiver, J.M., Dahoun, A. and Souahi, A. (1992) VideoControlled Tensile Testing of Polymers and Metals Beyond the Necking Point. J. Material Science, 27, 5031-5039.

G'Sell, C. (1995) Lois de comportement mécanique des polymères solides, in Introduction to Polymer Mechanics, INPL, 141-168.

Jarrin, J., Dewimille, B., Devaux, E. and Martin, J. (1994) Blistering of Thermoplastic Materials Used in the Petroleum Industry. 69th Annual Tech. Conf. and Exhibition of the Soc. of Petroleum Engineers, SPE 28482, 203-214.

Jolly, L. (2000) Analyse de la microstructure du polyamide 11 par diffusion des rayons $\mathrm{X}$. Application à une déformation uniaxiale. Thesis, University of Metz.

Loukil, M. (1996) Modélisation des surfaces de plasticité à partir d'une approche de la thermodynamique de la relaxation des milieux continus. Thesis, INPL.

Marchal, K. (1996) Influence du chemin de chargement sur le comportement du polyamide 11 autour de la transition vitreuse. Thesis, University of Poitiers.

Meimon, Y. (1990) Validation of Rheological Models for Typical Structures: An Attempt to Work out an Insurance Quality Procedure. Revue de l'Institut français du pétrole, 45, 525-541.

Meimon, Y. and Cangémi, L. (1999) Polymères semi-cristallins à usage structural : microstructures, comportement mécanique. Proc. of Colloque national Mecamat, 79-84

Pilvin, P. (1988) Identification des paramètres de modèles de comportement. Mecamat Symposium, Besançon, France, 155164.

Struik, L.C.E. (1987) The Mechanical and Physical Ageing of Semicrystalline Polymers: 1. Polymer, 28, 1521-1533.

Vincké, O. (1997) Essais de compression triaxiale sur du PA11 non plastifié. Private correspondence.

Wu, P. and Siesler, H.W. (1997) FT-NIR Spectroscopic Deuteration Studies and Rheo-Optical Investigations of Polyamide 11. IFP Research Report.

Final manuscript received in October 2000 